\title{
Las redes semánticas en torno a la burocracia y la función pública de los habitantes de Villahermosa, Tabasco, como parte de las representaciones sociales y la imagen de los servidores e instituciones públicas
}

The semantic networks around the bureaucracy and the public function
of the inhabitants of Villahermosa, Tabasco as part of the social
representations and the image of public servants and institutions

Arnulfo López Ramos*

Universidad Juárez Autónoma de Tabasco (UJAT)

arnulfo.lopezra@anahuac.mx

Av. Universidad $s / n$, Zona de la Cultura, Col. Magisterial,

C.P. 86040, Villahermosa, Tabasco, México

Raúl Santos Morales**

Universidad Anáhuac México

raul.santos@anahuac.mx

Av. Universidad Anáhuac núm. 46, Col. Lomas Anáhuac, C.P. 52786, Huixquilucan, Estado de México

Editor: Rogelio del Prado Flores

Fecha de recepción: 4 abril 2020

Fecha de aceptación: 9 mayo 2020

https://orcid.org/0000-0001-8974-6260

https://orcid.org/0000-0002-6128-9616

\section{RESUMEN}

A pesar de que la burocracia y la función pública son modelos, actuaciones y resultados de una forma muy específica de organización, trabajo y administración, inoperante para la mayor parte de la sociedad civil, las instituciones públicas que las practican generan una imagen institucional y diversas representaciones sociales que pueden ayudarnos a comprender las percepciones, aspiraciones, experiencias, juicios de valor y explicaciones, positivas o negativas, que las personas tienen al interactuar con ellas. Por lo que, a través de las redes semánticas naturales, se pudieron determinar los campos semánticos

* Licenciado en Comunicación, Maestro en Publicidad y Comunicación Corporativa. Doctorando en Investigación de la Comunicación en la Facultad de Comunicación de la Universidad Anáhuac México, Campus Norte.

** Licenciado en Publicidad, Maestro en Diseño Gráfico y Doctor en Comunicación Aplicada. Actualmente es profesor investigador del Centro de Investigación para la Comunicación Aplicada de la Universidad Anáhuac México. 
que hay sobre la burocracia y la función pública de una institución en Ioo personas de la ciudad de Villahermosa, Tabasco. Los resultados arrojan la coexistencia de valores positivos y negativos como parte de la imagen y de las representaciones sociales. De manera más específica, la burocracia tiene una imagen y representación social negativa, pero los funcionarios públicos tienen una imagen y representación social positiva. Un análisis más amplio de otras instituciones públicas establecerá un panorama más claro sobre los aspectos positivos o negativos de la imagen y de las representaciones sociales de las instituciones públicas de esa zona.

Palabras clave: Imagen, representaciones sociales, burocracia, función pública, instituciones públicas, comunicación organizacional.

\section{ABSTRACT}

Despite the fact that the bureaucracy and the public function are models, actions and results of a very specific form of organization, work and administration, ineffective for most of civil society, the public institutions that practice them generate an institutional image and various social representations that can help us understand the perceptions, aspirations, experiences, value judgments and explanations, positive or negative, that people have when interacting with them. Therefore, through the natural semantic networks, the semantic fields that exist about the bureaucracy and the public function of a public institution in Ioo people from the city of Villahermosa, Tabasco, could be determined. The results show the coexistence of positive and negative values as part of the image and social representations. More specifically, the bureaucracy has a negative image and social representation, but public officials have a positive imagen and social representation. A broader analysis of other public institutions will establish a clearer picture of the positive or negative aspects of the image and of the social representations of public institutions in that area.

Keywords: Image, social representations, bureaucracy, public function, public institutions, organizational communication.

\section{INTRODUCCIÓN}

T a burocracia es un fenómeno que ha sido estudiado desde muchas disciplinas, camLpos y áreas de estudio, por ejemplo, desde la sociología (Mouzelis, I973; Weber, I977; Clawson, I980; Barenstein, I982; Dandeker, I990; Lerner, I993; Du Gay, 2000;), desde la economía-política (Von Mises, I945; Jackson, I983; Page, I985; Leiby, I986; Savage, i995; 
Hunter, 1998; Du Gay, 2005; Richardson, 20II; Ahumada, Giacone y Hirtz, 20I4) desde la administración pública (Crozier, I964; Jenning, I966; Blau y Meyer, 1971; Carrasco Canals, I975; Hummel, I977; Arnold, I99I; INAP, 2005; Meier y Bohte, 2007; Byrkjeflot y Engelstad, 20I8; Carnevale y Stivers, 20I9), e incluso desde la filosofía (Eymar, 2009), el derecho (Meléndez George, 2005) y la historia (Cañas Gálvez, 2012), mientras que en el campo de la comunicación también existen investigaciones sobre la burocracia que se han centrado en la impersonalidad de las relaciones entre las personas y los servidores públicos (Lystad y Stone, 1956), en la anomia de las personas frente a las instituciones públicas y la burocracia (Marsh, Dolan y Riddick, 1967), en el lenguaje persuasivo utilizado por las personas ante los servidores públicos (Danet, I97I), en los diferentes tipos de comunicación burocrática (Rubin, 1974), en la comunicación interna dentro de las instituciones burocráticas (Bacharach y Aiken, 1977), en los entornos y la cultura organizacional de las instituciones públicas (Meyer, 1995), en los problemas burocráticos y la corrupción de grandes empresas (Picard, 1996), en la vinculación de las tecnologías de la información y la comunicación con la burocracia para generar procesos de transparencia (Snellen, 200I) o para que las instituciones públicas sean más funcionales (Bovens y Zouridis, 2002), en el reemplazo de la burocracia mediante las tecnologías de la información y comunicación (Harris, 2006; Ionescu, 20I6), en la mejora de los servicios de las instituciones burocráticas (Pärna y von Tunzelmann, 2007), en los procesos de innovación de las instituciones públicas (van Duivenboden y Thaens, 2008), en las dinámicas de interacción de las instituciones públicas para convertirlas en instituciones flexibles adaptadas al entorno (Klemsdal, 20I3), en la interacción entre la burocracia de las instituciones públicas y los medios respecto a la ayuda extranjera (Joly, 20I4), en la transición hacia el e-Gobierno mediante las tecnologías de información y comunicación (Buffat, 20I5), en los procesos burocráticos de comunicación interpersonal (Monteiro, 20I6), en el establecimiento de una agenda política a través de la burocracia (Baekgaard, Mortensen y Seeberg, 20I8) o en el uso de la política como parte de la comunicación burocrática (Ritchie, 20I8), así como en las experiencias burocráticas de comunicación interpersonal mediadas por las tecnologías de información y comunicación (Hansen, Lundberg y Syltevik, 20I8).

Sin embargo, ninguna de esas investigaciones ha tomado como punto de partida la imagen de una institución pública y su vinculación con las representaciones sociales como parte de un proceso de comunicación o interacción social. Aunque la burocracia es inoperante para la mayoría de las personas, es muy funcional para ciertos grupos privilegiados que la utilizan como una herramienta de poder, permitiéndoles establecer agendas políticas y económicas de control y dominación, además de obstaculizar la transparencia de la información y alcanzar una verdadera democracia (Baekgaard, Mortensen y Seeberg, 20I8; 
Ritchie, 20I8), por lo que seguir investigando sobre ella todavía es relevante para comprender otros aspectos que rodean a este modelo de organización, trabajo y administración de las instituciones públicas.

Entendemos por burocracia a un tipo de organización formal equipada para realizar tareas administrativas a gran escala de manera racional (DuBois y Berg, 2002), caracterizada por la lentitud, la ineficiencia y la impersonalidad (Gould y Howson, 20I8), debido al número de personas que intervienen en la cadena de transmisión de la información y al gran volumen de documentos que maneja (Coyne, 2008), ocasionando consecuencias negativas principalmente en la imagen, credibilidad y confianza de cualquier institución. Mientras que la función pública puede ser definida como la actuación y resultados del quehacer de las instituciones públicas vinculadas al Gobierno, a partir de un conjunto de elementos humanos que tratan de cumplir los compromisos y de resolver las eventualidades que se generan en las actividades gubernamentales para el servicio de la sociedad (Oropeza López, 2005).

La función pública y la burocracia forman parte de la imagen de una institución pública. Dentro de esta, la imagen son las percepciones, aspiraciones, experiencias y juicios de valor percibidas y generadas subjetivamente por las personas (Costa, 2009). La imagen entonces, se vincula directamente con la credibilidad y la confianza de lo que una institución dice que hace y de lo que las personas experimentan con ella, provocando una coherencia o incoherencia de las expectativas generadas, es decir, satisfacción, frustración o indiferencia hacia la institución (Costa, 2009), en este caso, como parte de un proceso de interacción entre diferentes grupos - servidores de las instituciones públicas y personas de la sociedad civil con obligaciones públicas- que ayudan a construir diversas representaciones sociales, entendidas como las explicaciones que las personas forman en sus relaciones cotidianas sobre un acontecimiento, hecho o fenómeno de naturaleza social, llamadas representaciones sociales, las cuales ayudan a elaborar los comportamientos y la comunicación entre las personas, permitiéndoles orientarse en un contexto social y material, además de otorgarles un entendimiento de sentido común a través de conceptos, enunciados y explicaciones como parte de la interacción social cotidiana (Moscovici, 1979, I98I).

Por lo tanto, para conocer el modo en el que una institución pública es representada y conocida por la sociedad, se recurre al concepto de representación social y a la imagen institucional donde ambas generan amplios universos de opinión (Moscovici, 1979) y coinciden a través del campo de representación que nos remite a la idea de imagen, de modelo social, a un contenido concreto y limitado de las proposiciones que se refieren a un aspecto parecido del objeto de la representación como los servidores públicos, lo que permite visualizar el carácter del contenido, las propiedades cualitativas o imaginativas, en un campo que inte- 
gra informaciones jerarquizadas a partir de fuentes inmediatas, en este caso, las personas (Moscovici, I979).

El análisis de las representaciones sociales permite conocer los juicios de valor sobre la imagen de una institución pública a partir de su elemento humano como los servidores públicos, lo que Moscovici (I979) llama campo de representación. Esto se logra mediante el uso de conceptos detonadores, en este caso, burocracia y función pública, lo que permite aproximarnos a los significados de manera directa con las personas (Valdez Medina, I998) y así conocer la interrelación o las conexiones que hay entre ambos conceptos mediante una red semántica (Tejada Tayabas y Arias Galicia, 2003), es decir, el conocimiento que existe sobre una institución pública a través de una serie de significados conectados o relacionados a través de palabras que varían entre sí, puesto que no todos los significados tienen el mismo peso debido a que las experiencias de vida son diversas, de la misma forma que lo son sus expectativas (Figueroa, González y Solís, I98I).

\section{MÉTODO Y DESARROLLO}

Como parte de un proyecto de investigación más amplio que trata de diseñar un modelo de comunicación organizacional para diversas dependencias de gobierno del Estado de Tabasco, México, de manera exploratoria, descriptiva y cuantitativa, se buscó conocer los juicios de valor positivos y/o negativos que existen alrededor de los servidores de una institución pública en la ciudad de Villahermosa, con la finalidad de comprender los significados que construyen la imagen actual de dicha institución y que, a la vez, forma parte de las representaciones sociales.

Por lo que durante el mes de octubre de 20I9, se realizó un análisis de significados a partir de la construcción de Redes Semánticas Naturales (RSN) de las palabras burocracia y función pública, utilizándolas como un estímulo que alude al objeto social a indagar, en este caso, a los servidores públicos que trabajan en las instituciones del Gobierno del Estado de Tabasco, por lo que se le pidió a roo ciudadanos de la ciudad de Villahermosa, capital de dicha entidad federativa, que realizaran una asociación libre para conformar una red de significados que ayudaran a construir la dimensión informativa de la representación social de las instituciones públicas a partir de los servidores públicos, analizando las correspondencias semánticas y estableciendo las similitudes o diferencias que existe entre los diversos campos obtenidos.

Esta técnica se vuelve mixta porque la elección de palabras es parte de un proceso individual y subjetivo mediante palabras estímulo otorgadas por el investigador y mencionadas por el sujeto al definir al objeto, conformando su carácter cualitativo, mientras que, al 
asignarle valores a lo observable cualitativamente para contar y medir cosas, esta técnica adquiere su carácter cuantitativo (Schwartz y Jacobs, 1984; Valdez Medina, 1998). En este caso, la investigación tiene un enfoque meramente cuantitativo.

La muestra estuvo constituida por 64 mujeres y 36 hombres de I 8 a 67 años, de los cuales más del $80 \%$ cuenta con estudios universitarios de licenciatura y posgrado, ya que se les considera con experiencia ciudadana en términos de interacción con el sistema burocrático de la función pública, a través de distintas instituciones de gobierno.

Para este estudio, se utilizaron palabras estímulo como burocracia y función pública a partir de las RSN para el análisis de correspondencia de los campos semánticos resultantes como componentes de ambos términos.

A través de un instrumento autoaplicable en formato electrónico, se les pidió a los sujetos de la muestra que mencionaran tres palabras que asocian con los términos detonadores burocracia y función pública; al mismo tiempo se les instruyó que las ordenasen de manera que en la primera posición del orden apareciera la palabra que mayor peso semántico consideran que tiene en relación con la palabra estímulo.

El instrumento estuvo conformado por dos secciones: con la primera parte se recabaron los datos sociodemográficos de los sujetos, tales como: género, edad, grado de escolaridad y actividad económica; mientras que en la segunda se abordó la indagación sobre las representaciones sociales, en cuyo primer ítem - para hacer un contraste posterior con el análisis final— se incluyeron 20 términos (ver Gráfica I) — Io positivos y Io negativossobre los cuales se le pidió a los sujetos de la muestra que seleccionaran libremente al menos tres, para determinar la calificación que le otorgan a los servidores públicos del gobierno de Tabasco.

Dichos términos fueron los siguientes:

- Términos positivos: Serviciales, ejemplares, honestos, trabajadores, eficientes, amables, educados, honrados, justos e insobornables.

- Términos negativos: Corruptos, groseros, lentos, desleales, flojos, prepotentes, ineficientes, ociosos, ignorantes e injustos.

Posteriormente, en el segundo y último ítem de esta sección se les pidió a los sujetos hacer una asociación libre a los términos principales de este estudio, y que al mismo tiempo ordenaran sus respuestas de acuerdo con el peso semántico que le asignaran a las palabras elegidas por ellos con autodeterminación.

Finalmente, se procesaron las respuestas con un procedimiento de tabulación de palabras definidoras (o generatrices) por peso semántico y frecuencia, a fin de lograr una pri- 
mera aproximación al significado de cada palabra detonadora. Los resultados se muestran a continuación.

\section{RESULTADOS}

Al procesar la información obtenida se encontraron asociados 88 términos generatrices para la RSN burocracia y Io8 para la R SN función pública. Para el análisis de los resultados, de acuerdo con el método explicado por José Luis Valdez Medina (I998), se seleccionaron las primeras I6 palabras con mayor peso semántico en cada RSN, mismas que dan la pauta para la discusión de resultados en este estudio exploratorio.

Con base a lo anterior, tal y como se muestra en las Gráficas 2 y 3, se constituyó el núcleo central de cada R SN, mismos que se consideran como el centro semántico del significado de cada término analizado, los cuales al ser contrastados con el resultado del primer ítem del instrumento donde se calificó a los servidores públicos de Tabasco, según los tabasqueños radicados en la ciudad de Villahermosa, permitieron realizar una aproximación más precisa sobre la representación social de las instituciones públicas en el sentir de los ciudadanos de dicha ciudad capital.

Al respecto, se tiene que los resultados de la primera parte del instrumento aplicado, en la que se le pidió a los entrevistados que calificaran a los servidores públicos en mención, indican que para estos los empleados de una institución pública del Gobierno del Estado de Tabasco son especialmente lentos, prepotentes, ineficientes y corruptos, tal y como se aprecia en la Gráfica I.

Al contrastar estos resultados con la técnica de RSN, se puede apreciar que existe una similitud del Iо०\% con el peso semántico que los entrevistados le asignaron al término lentitud que se muestra claramente en el conjunto SAM de la Gráfica 2, cuyo valor M, que indica el peso semántico total a partir de su jerarquía y frecuencia en el estudio, fue de I8I, de acuerdo con la tabulación de palabras definidoras que se exponen en la Tabla i que se muestra más adelante, representando así un valor FMG, el cual representa en términos de porcentajes la distancia semántica entre las palabras generatrices (Castañeda Morfín, 20I6), el IO०\% de la RSN burocracia, lo que la coloca en el centro del núcleo de la red semántica, seguida de otros términos como trámites, corrupción e ineficiencia, entre otros, con porcentajes FMG altos. 


\title{
GRÁFICA 1. RESULTADOS DE LA APROXIMACIÓN SEMÁNTICA SOBRE LOS SERVIDORES PÚBLICOS DEL GOBIERNO DE TABASCO
}

\begin{abstract}
Selecciona al menos tres de las siguientes palabras con las que calificarias a los servidores públicos del gobierno de Tabasco:
\end{abstract}

100 respuestas

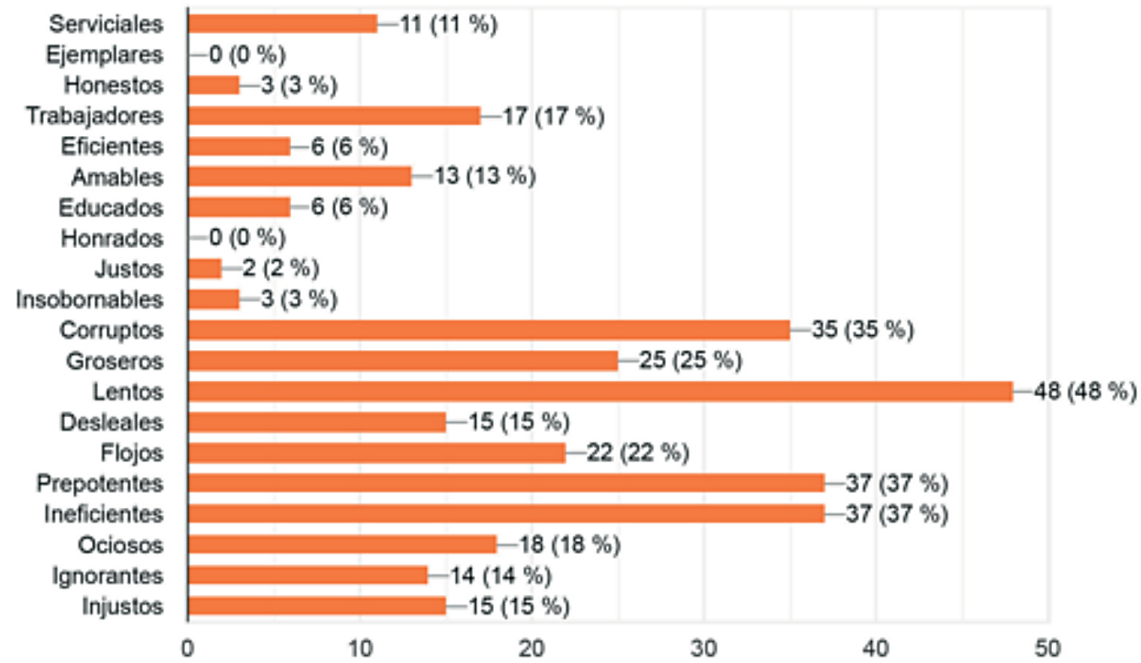

FUENTE: ELABORACIÓN PROPIA (2019).

\section{GRÁFICA 2. CONJUNTO SAM DE LA RED SEMÁNTICA BUROCRACIA}

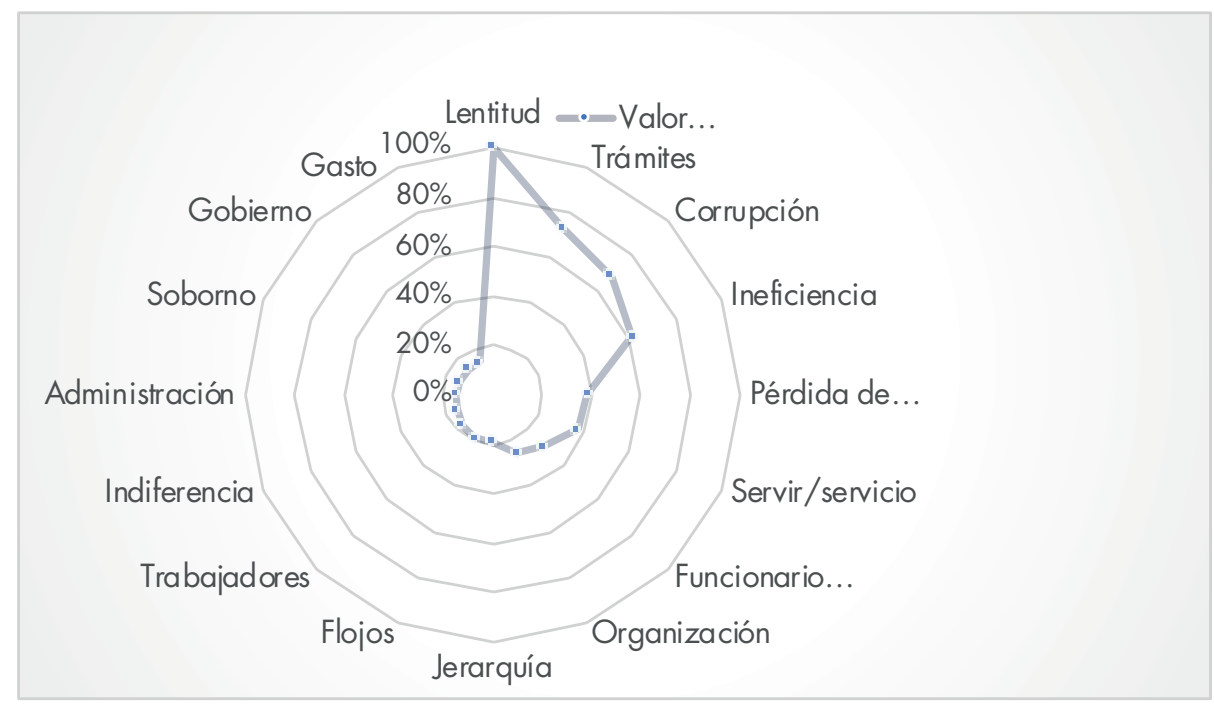

FUENTE: ELABORACIÓN PROPIA A PARTIR DE VALDEZ MEDINA (1998). 
Ahora bien, al revisar más a fondo en este punto de la investigación, los hallazgos desvelan el sentido de la percepción social registrada en el instrumento aplicado; al respecto, en un esfuerzo cualitativo por reconstituir gráficamente el sentir de los ciudadanos de Villahermosa, primero se realizó la tabulación de palabras definidoras del concepto burocracia (ver Tabla I).

TABLA 1. TABULACIÓN DE PALABRAS DEFINIDORAS DEL TÉRMINO BUROCRACIA, POR PESO SEMÁNTICO Y FRECUENCIA

\begin{tabular}{|c|c|c|c|c|c|c|}
\hline \multirow{2}{*}{$\begin{array}{c}\text { VALOR } \\
\mathrm{J}\end{array}$} & \multirow{2}{*}{ PALABRA DEFINIDORA } & \multicolumn{3}{|c|}{ FRECUENCIA } & \multirow{2}{*}{ VALOR M } & \multirow{2}{*}{ VALOR FMG } \\
\hline & & $1(10)$ & $2(6)$ & $3(3)$ & & \\
\hline 1. & Lentitud & $13(130)$ & $6(36)$ & $5(15)$ & 181 & $100 \%$ \\
\hline 2. & Trámites & $7(70)$ & $9(54)$ & $3(9)$ & 133 & $73.48 \%$ \\
\hline 3. & Corrupción & $8(80)$ & $5(30)$ & $4(12)$ & 122 & $67.40 \%$ \\
\hline 4. & Ineficiencia & $5(50)$ & $7(42)$ & $6(18)$ & 110 & $60.77 \%$ \\
\hline 5. & Pérdida de tiempo & $6(60)$ & $1(6)$ & $1(3)$ & 69 & $38.12 \%$ \\
\hline 6. & Servir/Servicio & $4(40)$ & $3(18)$ & $3(9)$ & 67 & $37.01 \%$ \\
\hline 7. & Funcionario público & $5(50)$ & & $1(3)$ & 53 & $29.28 \%$ \\
\hline 8. & Organización & $4(40)$ & $1(6)$ & & 46 & $25.41 \%$ \\
\hline 9. & Jerarquía & $2(20)$ & $1(6)$ & $3(9)$ & 35 & $19.33 \%$ \\
\hline 10. & Flojera/Flojos & $1(10)$ & $3(18)$ & $2(6)$ & 34 & $18.78 \%$ \\
\hline 11. & Trabajadores & $2(20)$ & $2(12)$ & & 32 & $17.67 \%$ \\
\hline 12. & Indiferencia & $2(20)$ & $1(6)$ & $1(3)$ & 29 & $16.02 \%$ \\
\hline 13. & Administración & & $4(24)$ & $1(3)$ & 27 & $14.91 \%$ \\
\hline 14. & Soborno & $2(20)$ & & $2(6)$ & 26 & $14.36 \%$ \\
\hline 15. & Gobierno & $2(20)$ & $1(6)$ & & 26 & $14.36 \%$ \\
\hline 16. & Gasto & $2(20)$ & $1(6)$ & & 26 & $14.36 \%$ \\
\hline 17. & Empleados & $1(10)$ & $2(12)$ & $1(3)$ & 25 & $13.81 \%$ \\
\hline 18. & Estancamiento & $1(10)$ & $2(12)$ & & 22 & $12.15 \%$ \\
\hline 19. & Política & $1(10)$ & $2(12)$ & & 22 & $12.15 \%$ \\
\hline
\end{tabular}




\begin{tabular}{|c|c|c|c|c|c|c|}
\hline \multirow{2}{*}{$\begin{array}{c}\text { VALOR } \\
\mathrm{J}\end{array}$} & \multirow{2}{*}{ PALABRA DEFINIDORA } & \multicolumn{3}{|c|}{ FRECUENCIA } & \multirow{2}{*}{ VALOR M } & \multirow{2}{*}{ VALOR FMG } \\
\hline & & $1(10)$ & $2(6)$ & $3(3)$ & & \\
\hline 20. & Anticuado & & $2(12)$ & $1(3)$ & 15 & $8.28 \%$ \\
\hline 21. & Gestión & $1(10)$ & & $1(3)$ & 13 & $7.18 \%$ \\
\hline 22. & Deficiencia & $1(10)$ & & $1(3)$ & 13 & $7.18 \%$ \\
\hline 23. & Actividad & $1(10)$ & & $1(3)$ & 13 & $7.18 \%$ \\
\hline 24. & Desigualdad & $1(10)$ & & $1(3)$ & 13 & $7.18 \%$ \\
\hline 25. & Servidor público & & $1(6)$ & $2(6)$ & 12 & $6.62 \%$ \\
\hline 26. & Educación & $1(10)$ & & & 10 & $5.52 \%$ \\
\hline 27. & Zalameros & $1(10)$ & & & 10 & $5.52 \%$ \\
\hline 28. & Burros & $1(10)$ & & & 10 & $5.52 \%$ \\
\hline 29. & Indolentes & $1(10)$ & & & 10 & $5.52 \%$ \\
\hline 30. & Manipulación & $1(10)$ & & & 10 & $5.52 \%$ \\
\hline 31. & Centralización & $1(10)$ & & & 10 & $5.52 \%$ \\
\hline 32. & Cargo & $1(10)$ & & & 10 & $5.52 \%$ \\
\hline 33. & Diplomacia & $1(10)$ & & & 10 & $5.52 \%$ \\
\hline 34. & Sindicatos & $1(10)$ & & & 10 & $5.52 \%$ \\
\hline 35. & Nepotismo & $1(10)$ & & & 10 & $5.52 \%$ \\
\hline 36. & Funcionamiento & $1(10)$ & & & 10 & $5.52 \%$ \\
\hline 37. & Inhumanos & $1(10)$ & & & 10 & $5.52 \%$ \\
\hline 38. & Intransigencia & $1(10)$ & & & 10 & $5.52 \%$ \\
\hline 39. & Amables & $1(10)$ & & & 10 & $5.52 \%$ \\
\hline 40. & Groseros & & $1(6)$ & $1(3)$ & 9 & $4.97 \%$ \\
\hline 41. & Demasiados & & $1(6)$ & $1(3)$ & 9 & $4.97 \%$ \\
\hline 42. & Orden & & $1(6)$ & $1(3)$ & 9 & $4.97 \%$ \\
\hline 43. & Incultos/lgnorancia & & $1(6)$ & $1(3)$ & 9 & $4.97 \%$ \\
\hline 44. & Compromiso & & $1(6)$ & $1(3)$ & 9 & $4.97 \%$ \\
\hline 45. & Malhumorados & & & $3(9)$ & 9 & $4.97 \%$ \\
\hline
\end{tabular}


LAS REDES SEMÁNTICAS EN TORNO A LA BUROCRACIA Y LA FUNCIÓN PÚBLICA DE LOS HABITANTES DE VILLAHERMOSA 113

\begin{tabular}{|c|c|c|c|c|c|c|}
\hline \multirow{2}{*}{$\begin{array}{c}\text { VALOR } \\
\mathrm{J}\end{array}$} & \multirow{2}{*}{ PALABRA DEFINIDORA } & \multicolumn{3}{|c|}{ FRECUENCIA } & \multirow{2}{*}{ VALOR M } & \multirow{2}{*}{ VALOR FMG } \\
\hline & & $1(10)$ & $2(6)$ & $3(3)$ & & \\
\hline 46. & Administrativos & & $1(6)$ & $1(3)$ & 9 & $4.97 \%$ \\
\hline 47. & Prepotencia & & $1(6)$ & $1(3)$ & 9 & $4.97 \%$ \\
\hline 48. & Deshonestos & & $1(6)$ & $1(3)$ & 9 & $4.97 \%$ \\
\hline 49. & Desleales & & $1(6)$ & & 6 & $3.31 \%$ \\
\hline 50. & Engorroso & & $1(6)$ & & 6 & $3.31 \%$ \\
\hline 51. & Seriedad & & $1(6)$ & & 6 & $3.31 \%$ \\
\hline 52. & Desconocimiento & & $1(6)$ & & 6 & $3.31 \%$ \\
\hline 53. & Dominio & & $1(6)$ & & 6 & $3.31 \%$ \\
\hline 54. & Injusticia & & & $2(6)$ & 6 & $3.31 \%$ \\
\hline 55. & Compadrazgo & & $1(6)$ & & 6 & $3.31 \%$ \\
\hline 56. & Robo & & $1(6)$ & & 6 & $3.31 \%$ \\
\hline 57. & Hombres & & $1(6)$ & & 6 & $3.31 \%$ \\
\hline 58. & Falsedad & & $1(6)$ & & 6 & $3.31 \%$ \\
\hline 59. & Torpes & & $1(6)$ & & 6 & $3.31 \%$ \\
\hline 60. & Frustración & & $1(6)$ & & 6 & $3.31 \%$ \\
\hline 61. & Descentralización & & $1(6)$ & & 6 & $3.31 \%$ \\
\hline 62. & Autoridad & & $1(6)$ & & 6 & $3.31 \%$ \\
\hline 63. & Participación & & $1(6)$ & & 6 & $3.31 \%$ \\
\hline 64. & Estado & & & $2(6)$ & 6 & $3.31 \%$ \\
\hline 65. & Complicaciones & & $1(6)$ & & 6 & $3.31 \%$ \\
\hline 66. & Calidad & & $1(6)$ & & 6 & $3.31 \%$ \\
\hline 67. & Inconformidad & & $1(6)$ & & 6 & $3.31 \%$ \\
\hline 68. & Falta de soluciones & & & $1(3)$ & 3 & $1.65 \%$ \\
\hline 69. & Cumplir & & & $1(3)$ & 3 & $1.65 \%$ \\
\hline 70. & Incorruptibles & & & $1(3)$ & 3 & $1.65 \%$ \\
\hline 71. & Poder & & & $1(3)$ & 3 & $1.65 \%$ \\
\hline
\end{tabular}




\begin{tabular}{|c|c|c|c|c|c|c|}
\hline \multirow{2}{*}{$\begin{array}{c}\text { VALOR } \\
\mathrm{J}\end{array}$} & \multirow{2}{*}{ PALABRA DEFINIDORA } & \multicolumn{3}{|c|}{ FRECUENCIA } & \multirow{2}{*}{ VALOR M } & \multirow{2}{*}{ VALOR FMG } \\
\hline & & $1(10)$ & $2(6)$ & $3(3)$ & & \\
\hline 72. & Padrinazgo & & & $1(3)$ & 3 & $1.65 \%$ \\
\hline 73. & Inercia & & & $1(3)$ & 3 & $1.65 \%$ \\
\hline 74. & Cansancio & & & $1(3)$ & 3 & $1.65 \%$ \\
\hline 75. & Conformista & & & $1(3)$ & 3 & $1.65 \%$ \\
\hline 76. & Desesperanza & & & $1(3)$ & 3 & $1.65 \%$ \\
\hline 77. & Carácter & & & $1(3)$ & 3 & $1.65 \%$ \\
\hline 78. & Arrogancia & & & $1(3)$ & 3 & $1.65 \%$ \\
\hline 79. & Beneficencia & & & $1(3)$ & 3 & $1.65 \%$ \\
\hline 80. & Responsabilidad & & & $1(3)$ & 3 & $1.65 \%$ \\
\hline 81. & Contabilidad & & & $1(3)$ & 3 & $1.65 \%$ \\
\hline 82. & Oficinas & & & $1(3)$ & 3 & $1.65 \%$ \\
\hline 83. & Manejable & & & $1(3)$ & 3 & $1.65 \%$ \\
\hline 84. & Tortura & & & $1(3)$ & 3 & $1.65 \%$ \\
\hline 85. & Conveniencia & & & $1(3)$ & 3 & $1.65 \%$ \\
\hline 86. & Prioridad & & & $1(3)$ & 3 & $1.65 \%$ \\
\hline 87. & Inconsistente & & & $1(3)$ & 3 & $1.65 \%$ \\
\hline 88. & Intolerables & & & $1(3)$ & 3 & $1.65 \%$ \\
\hline
\end{tabular}

FUENTE: ELABORACIÓN PROPIA A PARTIR DE VALDEZ MEDINA (1998).

Como se puede apreciar, en las valoraciones FMG más altas se posicionan palabras asociadas a la gestión de la burocracia en la que, evidentemente, se asocian los asuntos que conciernen al servicio público, por lo que resulta importante para el estudio una categorización por campo semántico para apreciar de mejor manera esta definición psicológica.

En este sentido, como resultado de la valoración de esta RSN se pudieron construir cinco Campos Semánticos (CS) que se relacionan directamente con el término burocracia. De tal manera que, haciendo un análisis conceptual de este caso, los 88 términos generatrices quedaron esquemáticamente agrupados en el mapa semántico respectivo de la siguiente manera: 
- CS Gestión: Desconocimiento, ineficiencia, lentitud, actividad, deficiencia, funcionamiento, inercia, trámites, pérdida de tiempo, frustración, engorroso, tortura, cansancio.

- CS Política: Desigualdad, estancamiento, manipulación, diplomacia, sindicatos, participación, Estado, inconformidad, seriedad, carácter, manejable.

- CS Empleados: Servidor público, funcionario público, demasiados, incorruptibles, trabajadores, burros, groseros, zalameros, flojos/flojera, malhumorados, intolerables, conformistas, indolentes, inhumanos, incultos/ignorantes, prepotentes, desleales, torpes, deshonestos, amables, arrogantes, inconscientes, indiferentes, intransigentes.

- CS Gobierno: Administración, corrupción, dominio, injusticia, servir/servicio, autoridad, nepotismo, robo, compadrazgo, soborno, padrinazgo, beneficencia, conveniencia, desesperanza, falta de soluciones, cumplir, prioridad.

- CS Organización: Jerarquía, anticuado, cargo, hombres, administrativos.

Estos cinco Cs, al momento de organizarlos dentro de un mapa semántico, agregan un valor interpretativo de la RSN que resulta importante en términos cualitativos, porque al hacer la revisión con esta perspectiva se puede encontrar sentido a las figuras simbólicas que los ciudadanos asocian con la burocracia.

Por ejemplo, el CS de Gestión, de acuerdo con lo que se aprecia en la Figura I, explica de alguna manera cómo se constituyen estas figuras simbólicas en el colectivo social que interactúa con las instituciones de gobierno. En este caso destaca la importancia que los ciudadanos otorgan al sentido del tiempo cuando acuden a las dependencias públicas por algún servicio en especial; ellos consideran que la gestión burocrática no solo es lenta, sino que implica para ellos trámites engorrosos, pérdida de tiempo y frustración, principalmente. Sin embargo, la percepción que se tiene sobre las personas que trabajan para el Estado es de connotaciones más negativas que positivas (ver Figura i).

Por su parte, en lo que corresponde al término función pública, entre los hallazgos principales destacan desde el contraste con la primera valoración hecha sobre la aproximación semántica en torno de los servidores públicos de Tabasco que se muestran en los io8 términos generatrices agrupados en la Tabla 2, similitudes en cuanto a los términos corrupción e ineficiencia, cuyo valor FMG supera el 6r y 39\%, respectivamente, dentro del núcleo de la segunda RSN de este estudio (Gráfica 3). 
FIGURA 1. MAPA SEMÁNTICO DEL TÉRMINO BUROCRACIA

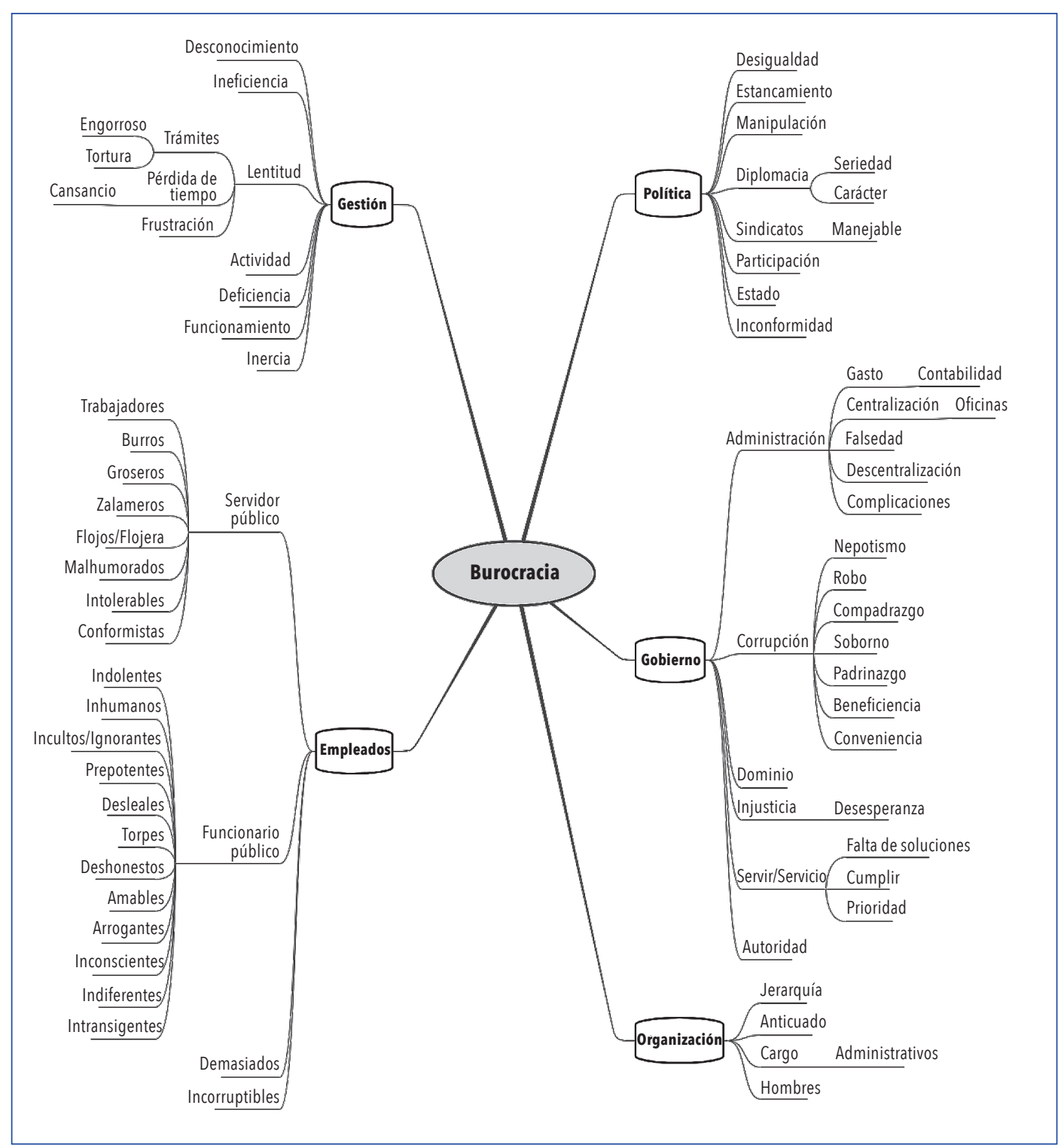

FUENTE: ELABORACIÓN PROPIA (2019). 
GRÁFICA 3. CONJUNTO SAM DE LA RED SEMÁNTICA FUNCIÓN PÚBLICA

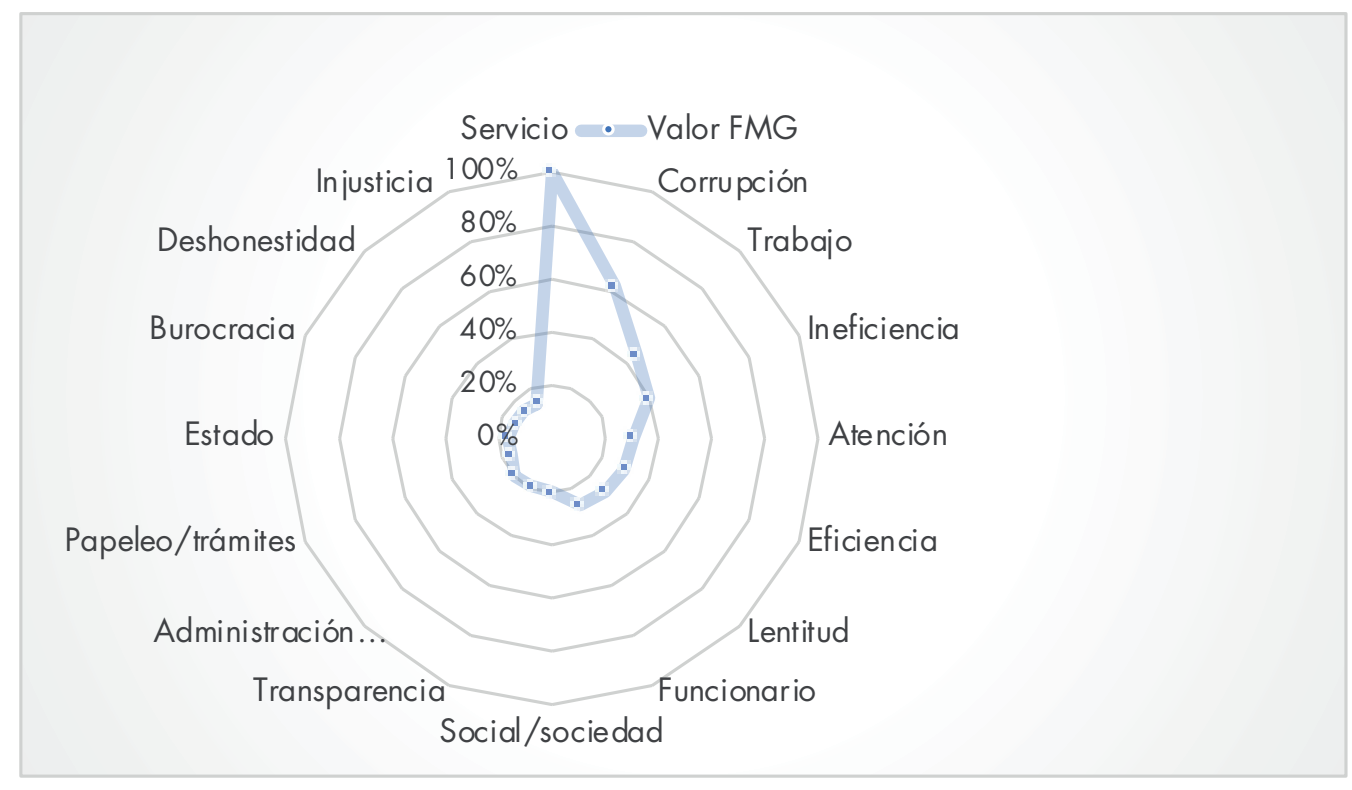

FUENTE: ELABORACIÓN PROPIA A PARTIR DE VALDEZ MEDINA (1998).

De igual manera, en cuanto a la construcción de los CS con la misma técnica de RSN, fue posible asociar las palabras generatrices en siete campos semánticos, donde es clara la asociación que los entrevistados hacen del término gobierno como el componente principal de la RS que alude a los asuntos que tienen que ver con el servicio público: la corrupción, transparencia en el ejercicio, la burocracia, la injusticia y el poder, entre otros términos (ver Figura 2).

TABLA 2. TABULACIÓN DE PALABRAS DEFINIDORAS DEL TÉRMINO

FUNCIÓN PÚBLICA, POR PESO SEMÁNTICO Y FRECUENCIA

\begin{tabular}{c|l|r|r|r|r|r|}
$\begin{array}{c}\text { VALOR } \\
\text { J }\end{array}$ & \multicolumn{1}{c}{ PALABRA DEFINIDORA } & $1(10)$ & $2(6)$ & $3(3)$ & VALOR M & VALOR FMG \\
\hline 1 & Servicio & $12(120)$ & $6(36)$ & $7(21)$ & 177 & $100 \%$ \\
\hline 2 & Corrupción & $7(70)$ & $5(30)$ & $3(9)$ & 109 & $61.58 \%$ \\
\hline 3 & Trabajo & $7(70)$ & $1(6)$ & $1(3)$ & 79 & $44.63 \%$ \\
\hline 4 & Ineficiencia & $4(40)$ & $2(12)$ & $6(18)$ & 70 & $39.54 \%$ \\
\hline
\end{tabular}




\begin{tabular}{|c|c|c|c|c|c|c|}
\hline \multirow{2}{*}{$\underset{\mathrm{J}}{\mathrm{VALOR}}$} & \multicolumn{6}{|c|}{ FRECUENCIA } \\
\hline & PALABRA DEFINIDORA & $1(10)$ & $2(6)$ & $3(3)$ & VALOR M & VALOR FMG \\
\hline 5 & Atención & $3(30)$ & $3(18)$ & $2(6)$ & 54 & $30.50 \%$ \\
\hline 6 & Eficaz/Eficiencia & $2(20)$ & $4(24)$ & $3(9)$ & 53 & $29.94 \%$ \\
\hline 7 & Lentitud & $2(20)$ & $4(24)$ & $2(6)$ & 50 & $28.24 \%$ \\
\hline 8 & Funcionario & $3(30)$ & $3(18)$ & & 48 & $27.11 \%$ \\
\hline 9 & Social/Sociedad & $3(30)$ & $1(6)$ & & 36 & $20.33 \%$ \\
\hline 10 & Transparencia & $2(20)$ & $1(6)$ & $3(9)$ & 35 & $19.77 \%$ \\
\hline 11 & Administración pública & $2(20)$ & $2(12)$ & $1(3)$ & 35 & $19.77 \%$ \\
\hline 12 & Papeleo/Trámites & $2(20)$ & $1(6)$ & $1(3)$ & 29 & $16.38 \%$ \\
\hline 13 & Estado & $2(20)$ & $1(6)$ & $1(3)$ & 29 & $16.38 \%$ \\
\hline 14 & Burocracia & $2(20)$ & $1(6)$ & & 26 & $14.68 \%$ \\
\hline 15 & Deshonestidad & $1(10)$ & $1(6)$ & $3(9)$ & 25 & $14.12 \%$ \\
\hline 16 & Injusticia & $1(10)$ & $2(12)$ & $1(3)$ & 25 & $14.12 \%$ \\
\hline 17 & Poder & $2(20)$ & & $1(3)$ & 23 & $12.99 \%$ \\
\hline 18 & Responsabilidad & $2(20)$ & & & 20 & $11.29 \%$ \\
\hline 19 & Políticos & $1(10)$ & $1(6)$ & $1(3)$ & 19 & $10.73 \%$ \\
\hline 20 & Ignorancia & $1(10)$ & $1(6)$ & $1(3)$ & 19 & $10.73 \%$ \\
\hline 21 & Soborno & & $2(12)$ & $2(6)$ & 18 & $10.16 \%$ \\
\hline 22 & Deslealtad & $1(10)$ & & $2(6)$ & 16 & $9.03 \%$ \\
\hline 23 & Ayuda & $1(10)$ & $1(6)$ & & 16 & $9.03 \%$ \\
\hline 24 & Apoyo & $1(10)$ & $1(6)$ & & 16 & $9.03 \%$ \\
\hline 25 & Respeto & $1(10)$ & $1(6)$ & & 16 & $9.03 \%$ \\
\hline 26 & Honestidad & & & $5(15)$ & 15 & $8.47 \%$ \\
\hline 27 & Indiferencia & $1(10)$ & & $1(3)$ & 13 & $7.34 \%$ \\
\hline 28 & Empleados & & $2(12)$ & & 12 & $6.77 \%$ \\
\hline 29 & Arrogancia & & $2(12)$ & & 12 & $6.77 \%$ \\
\hline 30 & Abuso del cargo & $1(10)$ & & & 10 & $5.64 \%$ \\
\hline 31 & Simulación & $1(10)$ & & & 10 & $5.64 \%$ \\
\hline
\end{tabular}




\begin{tabular}{|c|c|c|c|c|c|c|}
\hline \multirow{2}{*}{ VALOR } & \multicolumn{6}{|c|}{ FRECUENCIA } \\
\hline & & & & & & \\
\hline $\mathrm{J}$ & PALABRA DEFINIDORA & $1(10)$ & $2(6)$ & $3(3)$ & VALOR M & VALOR FMG \\
\hline 32 & Tapadera & $1(10)$ & & & 10 & $5.64 \%$ \\
\hline 33 & Secretaría & $1(10)$ & & & 10 & $5.64 \%$ \\
\hline 34 & Tardanza & $1(10)$ & & & 10 & $5.64 \%$ \\
\hline 35 & Organización & $1(10)$ & & & 10 & $5.64 \%$ \\
\hline 36 & Ágil & $1(10)$ & & & 10 & $5.64 \%$ \\
\hline 37 & Mentiras & $1(10)$ & & & 10 & $5.64 \%$ \\
\hline 38 & Oportunidad & $1(10)$ & & & 10 & $5.64 \%$ \\
\hline 39 & Groseros & $1(10)$ & & & 10 & $5.64 \%$ \\
\hline 40 & Desorden & $1(10)$ & & & 10 & $5.64 \%$ \\
\hline 41 & Nepotismo & $1(10)$ & & & 10 & $5.64 \%$ \\
\hline 42 & Resultados & $1(10)$ & & & 10 & $5.64 \%$ \\
\hline 43 & Inminente & $1(10)$ & & & 10 & $5.64 \%$ \\
\hline 44 & $\begin{array}{l}\text { Vigilar actividades } \\
\text { públicas }\end{array}$ & $1(10)$ & & & 10 & $5.64 \%$ \\
\hline 45 & Dependencia & $1(10)$ & & & 10 & $5.64 \%$ \\
\hline 46 & Información & $1(10)$ & & & 10 & $5.64 \%$ \\
\hline 47 & Seguridad & $1(10)$ & & & 10 & $5.64 \%$ \\
\hline 48 & Intolerancia & $1(10)$ & & & 10 & $5.64 \%$ \\
\hline 49 & Élite & $1(10)$ & & & 10 & $5.64 \%$ \\
\hline 50 & Ministros & $1(10)$ & & & 10 & $5.64 \%$ \\
\hline 51 & Irresponsabilidad & $1(10)$ & & & 10 & $5.64 \%$ \\
\hline 52 & Gobierno & $1(10)$ & & & 10 & $5.64 \%$ \\
\hline 53 & Engreídos & $1(10)$ & & & 10 & $5.64 \%$ \\
\hline 54 & Desconfianza & $1(10)$ & & & 10 & $5.64 \%$ \\
\hline 55 & Conjunto & $1(10)$ & & & 10 & $5.64 \%$ \\
\hline 56 & Calidad & $1(10)$ & & & 10 & $5.64 \%$ \\
\hline 57 & Necesaria & $1(10)$ & & & 10 & $5.64 \%$ \\
\hline
\end{tabular}




\begin{tabular}{|c|c|c|c|c|c|c|}
\hline \multirow{2}{*}{$\begin{array}{c}\text { VALOR } \\
\mathrm{J}\end{array}$} & \multicolumn{6}{|c|}{ FRECUENCIA } \\
\hline & PALABRA DEFINIDORA & $1(10)$ & $2(6)$ & $3(3)$ & VALOR M & VALOR FMG \\
\hline 58 & Gratuidad & $1(10)$ & & & 10 & $5.64 \%$ \\
\hline 59 & Compromiso & & $1(6)$ & $1(3)$ & 9 & $5.08 \%$ \\
\hline 60 & Conveniencia & & $1(6)$ & $1(3)$ & 9 & $5.08 \%$ \\
\hline 61 & Normatividad & & $1(6)$ & $1(3)$ & 9 & $5.08 \%$ \\
\hline 62 & Solución & & $1(6)$ & $1(3)$ & 9 & $5.08 \%$ \\
\hline 63 & Prepotencia & & $1(6)$ & $1(3)$ & 9 & $5.08 \%$ \\
\hline 64 & Igualdad & & $1(6)$ & $1(3)$ & 9 & $5.08 \%$ \\
\hline 65 & Iniciativa & & $1(6)$ & & 6 & $3.38 \%$ \\
\hline 66 & Amables & & & $2(6)$ & 6 & $3.38 \%$ \\
\hline 67 & Recursos & & $1(6)$ & & 6 & $3.38 \%$ \\
\hline 68 & Flojos & & $1(6)$ & & 6 & $3.38 \%$ \\
\hline 69 & Procedimientos & & $1(6)$ & & 6 & $3.38 \%$ \\
\hline 70 & Escalón & & $1(6)$ & & 6 & $3.38 \%$ \\
\hline 71 & Retraso & & $1(6)$ & & 6 & $3.38 \%$ \\
\hline 72 & Honradez & & $1(6)$ & & 6 & $3.38 \%$ \\
\hline 73 & Dinero/Economía & & & $2(6)$ & 6 & $3.38 \%$ \\
\hline 74 & Obligación & & $1(6)$ & & 6 & $3.38 \%$ \\
\hline 75 & Institucionalidad & & $1(6)$ & & 6 & $3.38 \%$ \\
\hline 76 & Oficinas & & $1(6)$ & & 6 & $3.38 \%$ \\
\hline 77 & Protección & & $1(6)$ & & 6 & $3.38 \%$ \\
\hline 78 & Inteligente & & $1(6)$ & & 6 & $3.38 \%$ \\
\hline 79 & Problemas & & $1(6)$ & & 6 & $3.38 \%$ \\
\hline 80 & Desarrollo & & $1(6)$ & & 6 & $3.38 \%$ \\
\hline 81 & Amistad & & $1(6)$ & & 6 & $3.38 \%$ \\
\hline 82 & Empatía & & $1(6)$ & & 6 & $3.38 \%$ \\
\hline 83 & Frustrante & & $1(6)$ & & 6 & $3.38 \%$ \\
\hline 84 & Gobernados & & $1(6)$ & & 6 & $3.38 \%$ \\
\hline
\end{tabular}


LAS REDES SEMÁNTICAS EN TORNO A LA BUROCRACIA Y LA FUNCIÓN PÚBLICA DE LOS HABITANTES DE VILLAHERMOSA 121

\begin{tabular}{|c|c|c|c|c|c|c|}
\hline \multirow{2}{*}{$\begin{array}{c}\text { VALOR } \\
\mathrm{J}\end{array}$} & \multicolumn{6}{|c|}{ FRECUENCIA } \\
\hline & PALABRA DEFINIDORA & $1(10)$ & $2(6)$ & $3(3)$ & VALOR M & VALOR FMG \\
\hline 85 & Pueblo & & $1(6)$ & & 6 & $3.38 \%$ \\
\hline 86 & Ley & & $1(6)$ & & 6 & $3.38 \%$ \\
\hline 87 & Impunidad & & $1(6)$ & & 6 & $3.38 \%$ \\
\hline 88 & Incumplimiento & & $1(6)$ & & 6 & $3.38 \%$ \\
\hline 89 & Inútil & & & $1(3)$ & 3 & $1.69 \%$ \\
\hline 90 & Ética & & & $1(3)$ & 3 & $1.69 \%$ \\
\hline 91 & Jefe & & & $1(3)$ & 3 & $1.69 \%$ \\
\hline 92 & Control & & & $1(3)$ & 3 & $1.69 \%$ \\
\hline 93 & Arcaica & & & $1(3)$ & 3 & $1.69 \%$ \\
\hline 94 & Obsoleta & & & $1(3)$ & 3 & $1.69 \%$ \\
\hline 95 & Demandante & & & $1(3)$ & 3 & $1.69 \%$ \\
\hline 96 & Soberbia & & & $1(3)$ & 3 & $1.69 \%$ \\
\hline 97 & Competitividad & & & $1(3)$ & 3 & $1.69 \%$ \\
\hline 98 & Ociosidad & & & $1(3)$ & 3 & $1.69 \%$ \\
\hline 99 & Datos & & & $1(3)$ & 3 & $1.69 \%$ \\
\hline 100 & Hipócrita & & & $1(3)$ & 3 & $1.69 \%$ \\
\hline 101 & Lealtad & & & $1(3)$ & 3 & $1.69 \%$ \\
\hline 102 & Ineptitud & & & $1(3)$ & 3 & $1.69 \%$ \\
\hline 103 & Participación & & & $1(3)$ & 3 & $1.69 \%$ \\
\hline 104 & Fracaso & & & $1(3)$ & 3 & $1.69 \%$ \\
\hline 105 & Sobrevaluada & & & $1(3)$ & 3 & $1.69 \%$ \\
\hline 106 & Aclaraciones & & & $1(3)$ & 3 & $1.69 \%$ \\
\hline 107 & Sanción & & & $1(3)$ & 3 & $1.69 \%$ \\
\hline 108 & Valores & & & $1(3)$ & 3 & $1.69 \%$ \\
\hline
\end{tabular}

FUENTE: ELABORACIÓN PROPIA A PARTIR DE VALDEZ MEDINA (1998). 
En este sentido, del análisis conceptual correspondiente a estos Io 8 términos generatrices en este caso, esquemáticamente resultaron agrupados en siete campos dentro del mapa semántico de la siguiente manera:

- CS Gobierno: Servicio, corrupción, transparencia, burocracia, administración pública, injusticia, poder, responsabilidad, seguridad, iniciativa, gobernados, ayuda, apoyo, calidad, gratuidad, solución, competitividad, soborno, tapadera, nepotismo, conveniencia, amistad, simulación, mentiras, información, datos, aclaraciones, eficiencia, ineficiencia, atención, ágil, lentitud, papeleo, tardanza, frustrante, valores, resultados, compromiso, incumplimiento, recursos, dinero, procedimientos, desarrollo, fracaso, problemas, impunidad, inminente, élite, respeto.

- CS Política: Sociedad, desconfianza, igualdad, pueblo, participación, demandante.

- CS Empleados: Trabajo, funcionarios, ignorantes, deslealtad, indiferencia, arrogancia, engreídos, abuso del cargo, escalón, groseros, ministros, irresponsabilidad, prepotencia, soberbia, jefe, ineptitud, deshonestidad, hipocresía, honestidad, honradez, intolerancia, amables, empatía, flojos, ociosidad, inteligente.

- CS Arcaica: Inútil, obsoleta.

- CS Necesaria: Conjunto, lealtad, sobrevaluada.

- CS Estado: Vigilar actividades públicas, normatividad, obligación, institucionalidad, protección, ética.

- CS Organización: Secretaría, dependencia, oficinas, oportunidad, desorden, retraso, ley, control, sanción.

La representación gráfica de estos siete campos se muestra en el siguiente mapa semántico: 
FIGURA 2. MAPA SEMÁNTICO DEL TÉRMINO FUNCIÓN PÚBLICA

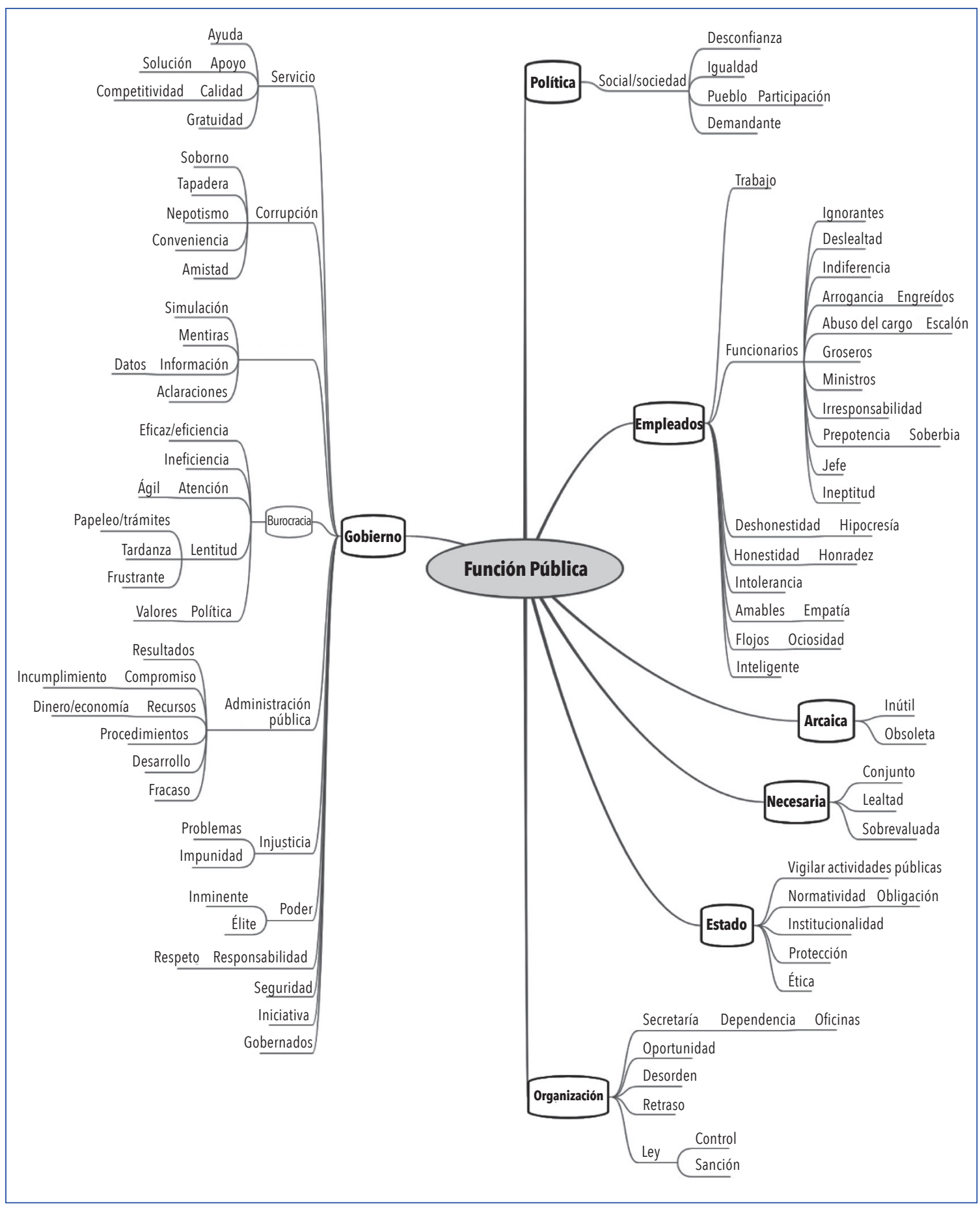




\section{CONCLUSIONES}

Los resultados indican que existen juicios de valor positivo y negativo coexistentes entre sí. Bajo el concepto de burocracia, existen más aspectos negativos que positivos, mientras que en el concepto de función pública es a la inversa, donde los aspectos positivos superan a los aspectos negativos.

Esta ambivalencia muestra, de manera no concluyente, que los servidores públicos tienen una imagen y una representación social negativa en sus procesos de organización, trabajo y administración — burocracia-, pero al ser elementos humanos, sus actuaciones y resultados — función pública — tienen una imagen y representación positiva, lo que de alguna manera sigue comprobando la lucha de percepciones, aspiraciones, experiencias, juicios de valor y explicaciones a las que se enfrentan las instituciones públicas por parte de las personas, por lo que un análisis más amplio y diverso dentro del Estado de Tabasco permitirá establecer un panorama más claro sobre los aspectos positivos o negativos de la imagen y de las representaciones sociales de las instituciones públicas de esa zona.

\section{REFERENCIAS}

Ahumada, J., Giacone, M. y Hirtz, N. (2OI4). Estudios sobre burocracia, estado y capitalismo. Argentina: Brujas.

Arnold, L. (I99I). Burocracia y burócratas en México 1742-I835. México: Consejo Nacional para la Cultura y las Artes.

Bacharach, S. B. y Aiken, M. (1977). Communication in administrative bureaucracies. Academy of Management Fournal, 2O(3),365-377. https://doi.org/10.2307/255411

Baekgaard, M., Mortensen, P. B. y Seeberg, H. B. (2018). The bureaucracy and the policy agenda. Fournal of Public Administration Research \&oTheory,28(2), 239-253.https://doi.org/10.1093/ jopart/mux045

Barenstein, J. (1982). El análisis de la burocracia estatal desde la perspectiva weberiana. 2a. ed. México: CIDE.

Blau, P. M. y Meyer, M. W. (I97I). Bureaucracy in modern society. 2a. ed. New York: Random-House.

Bovens, M. y Zouridis, S. (2002). From street-level to system-level bureaucracies: how information and communication technology is transforming administrative discretion and constitutional control. Public Administration Review, 62(2), I74. https://doi.org/10.1111/0033$\underline{3352.00168}$

Buffat, A. (2015). Street-level bureaucracy and e-government. Public Management Review, I7(I), I49-I6r. https://doi.org/10.1080/14719037.2013.771699 
Byrkjeflot, H. y Engelstad, F. (2018). Bureaucracy and society in transition: comparative perspectives. Bingley, UK: Emerald.

Cañas, F. D. P. (2012). Burocracia y cancillería en la Corte de Fuan II de Castilla (I406-I454): estudio institucional y proposográfico. Salamanca, España: Ediciones Universidad de Salamanca.

Carnevale, D. G. y Stivers, C. (2019). Knowledge and power in public bureaucracies. From pyramid to circle. New York: Routledge.

Carrasco, C. (1975). La burocracia en la España del siglo XIX. Madrid, España: Instituto de Estudios de Administración Local.

Castañeda, A. (20I6). Las redes semánticas naturales como estrategia metodológica para conocer las representaciones sociales acerca de la investigación en el contexto de la formación profesional de los comunicadores. Estudios sobre las culturas contemporáneas, I2(43), I23-I68.

Clawson, D. (1980). Bureaucracy and the labor process: the transformation of U.S. industry, I860-1920. New York: Monthly Bureaucracy Press.

Costa,J. (2009).ElDirCom hoy. Direccióny gestión de la comunicación en la nueva economía. Barcelona, España: Costa Punto Com.

Coyne, C. J. (2008). The politics of bureaucracy and the failure of postwar reconstruction. Public Choice, I35: II-22.

Crozier, M. (1964). The bureaucratic phenomenon. Chicago, IL: University of Chicago Press.

Dandeker, C. (1990). Surveillance, power and modernity: bureaucracy and discipline from I700 to present day. Cambridge, UK: Polity Press.

Danet, B. (1971). The language of persuasion in bureaucracy: "modern" and "traditional" appeals to the Israel customs authorities. American Sociological Review, 36(5), 847-859. https://doi. org $/ 10.2307 / 2093671$

Du Gay, P. (2000). In praise of bureaucracy: Weber, organization and etbics. London, UK: Sage.

Du Gay, P. (ed). (2005). The values of bureaucracy. Oxford, UK: Oxford University Press.

Eymar, C. (2009). El funcionario poeta: elementos para una estética de la burocracia. Madrid, España: Fórcola Ediciones.

Figueroa, J. G., González, E. y Solís, V. (198I). Una aproximación al problema del significado: las redes semánticas. Revista Latinoamericana de Psicología, I3(3), 447-458.

Gould, M. y Howson, A. (2018). Bureaucratic surveillance. Social interactions in groups and organizations. Research Starters Sociology, I-6.

Hansen, H., Lundberg, K. y Syltevik, L. J. (20I8). Digitalization, street-level bureaucracy and welfare users' experiences. Social Policy \&ٌ Administration, 52(I), 67-90. https://doi.org/10.1111/ spol.12283

Harris, M. (2006). Technology, innovation and post-bureaucracy: the case of the British Library. Fournal of Organizational Change Management, I9(I), 80-92. https://doi.org/10.1108/ 09534810610643703

Hummel, R. P. (1977). The bureaucratic experience. New York: St. Martin’s Press. 
Hunter, I. (1998). Repensar la escuela: subjetividad, burocracia y crítica. Barcelona, España: Pomares-Corredor.

Instituto Nacional de Administración Pública. (2005). De la burocracia al management. Del management a la gobernanza. Madrid, España: Instituto Nacional de Administración Pública.

Ionescu, L. (2016). Bureaucratic communication in government administration. Annals of Spiru Haret University, Fournalism Studies, I7(I), 5-I2.

Jackson, P. M. (1983). The political economy of bureaucracy. Totowa, NJ: Barnes \& Noble.

Jenning, E. E. (1966). El ejecutivo en la empresa y en la administración pública: autócrata, burócrata, demócrata. Barcelona, España: Sagitario.

Joly, J. (2014). Do the media influence foreign aid because or in spite of the bureaucracy? A case study of Belgian aid determinants. Political Communication, $3 I(4), 584-603 . \underline{\text { https://doi.org/10.108 }}$ 0/10584609.2013.879361

Klemsdal, L. (2013). From bureaucracy to learning organization: critical minimum specification design as space for sensemaking. Systemic Practice \&o Action Research, 26(I), 39-52. https://doi.org/10.1007/s11213-012-9267-3

Leiby, J. S. (1986). Colonial bureaucrats and the Mexican economy growth of a patrimonial state. I763I82I. New York: P. Lang.

Lerner, B. (1993).Democracia política o dictadura de las burocracias. Una lectura de Max Weber con miras al porvenir. México: UNAM.

Lystad, M. H. y Stone, R. C. (1956). Bureaucratic mass media: a study in role definitions. Social Forces, 34(4), 356-36r. https://doi.org/10.2307/2573670

Marsh, C. P., Dolan, R. J. y Riddick, W. L. (1967). Anomia and communication behavior: the relationship between anomia and utilization of three public bureaucracies. Rural Sociology, 32 (4), 435-445.

Meier, K. J. y Bohte, J. (2007). Politics and the bureaucracy. Policymaking in the fourth branch of government. Belmont, CA: Wadsworth Cengage Learning.

Meléndez, L. (2005). Derecho burocrático (incertidumbre jurídica). México: Porrúa.

Meyer, H. D. (1995). Organizational environments and organizational discourse: bureaucracy between two worlds. Organization Science, 6(I), 32-43. https://doi.org/10.1287/orsc.6.1.3

Monteiro, D. (2016). Street-level bureaucracy revisited. Language \& Dialogue, 6(I), 54-80. https://doi.org/10.1075/ld.6.1.02mon

Moscovici, S. (I98I). On social representations. En J. P. Forgas (ed). Social cognition perspectives on everyday knowledge (pp. I8I-209). London, UK: Academic Press.

Moscovici, S. (ed.) (1985). Psicología social I. Influencia y cambio de actitudes, individuos y grupos. Madrid, España: Paidós.

Mouzelis, N.P.(1973). Organizacióny burocracia: un análisis de las teorías modernas sobre organizaciones sociales. Barcelona, España: Península.

Oropeza, A. (2005). La evaluación de la función pública en México. México: Plaza y Valdés. 
Page, E. C. (1985). Political autbority and bureaucratic power: a comparative analysis. Knoxville, TN: University of Tennesse Press.

Pärna, O. y von Tunzelmann, N. (2007). Innovation in the public sector: Key features influencing the development and implementation of technologically innovative public sector services in the UK, Denmark, Finland and Estonia. Information Polity: The International Fournal of Government \& Democracy in the Information Age, I2(3), I09-I25. https://doi.org/10.3233/IP-2007-0118

Picard, R. G. (1996). The rise and fall of communication empires. Fournal of Media Economics, 9(4), 23. https://doi.org/10.1207/s15327736me0904_2

Richardson, S. (2OII). The political economy of bureaucracy. Oxford, UK: Routledge.

Ritchie, M. N. (20I8). Back-channel representation: a study of the strategic communication of senators with the US Department of Labor. Fournal of Politics, 80(I), 240-253. https://doi.org/10.1086/694395

Rubin, H. J. (1974). Modes of bureaucratic communications: examples from Thai local administration. Sociological Quarterly, I5(2), 2I2-230. https://doi.org/10.1111/j.1533-8525.1974. tb00889.x

Savage, M. (1995). Property, bureucracy, and culture: middle-class formation in contemporary Britain. London, UK: Routledge.

Schwartz, H. y Jacobs, J. (1984). Sociología cualitativa. México: Trillas.

Snellen, I. (200I). ICTs, bureaucracies, and the future of democracy. Communications of the ACM, 44(I), 45-48. https://doi.org/10.1145/357489.357504

Tejada, J. M. y Arias, L. F. (2003). El significado de tutoría académica en estudiantes de primer ingreso a la licenciatura. Revista de la Educación Superior, 32(127), 25-38.

Valdez, J. L. (1998). Las redes semánticas naturales, usos y aplicaciones en psicología social. Universidad Autónoma del Estado de México.

Van Duivenboden, H., y Thaens, M. (2008). ICT-driven innovation and the culture of public administration: A contradiction in terms? Information Polity: The International Fournal of Government \& Democracy in the Information Age, I3 (3/4), 213-232. https://doi.org/10.3233/IP-2008-0157

Von Mises, L. (1945). Bureaucracy. Glasgow, Scotland: William Hodge \& Co.

Weber, M. (1977).¿Qué es la burocracia? Buenos Aires, Argentina: Pléyade. 